\title{
Bridged bicyclic building block upgrading: Photochemical synthesis of bicyclo[3.1.1] heptan-1-amines
}

Alexander S. Harmata, Madison J. Sowden, Corey R. J. Stephenson*

Willard Henry Dow Laboratory, Department of Chemistry, University of Michigan, 930 North University Avenue, Ann Arbor, Michigan 48109, United States.

*Correspondence to: crjsteph@umich.edu

ABSTRACT: Compounds containing bridged bicyclic carbon skeletons are desirable building blocks for medicinal chemistry. However, as a result of their inefficient, linear syntheses, commercially available compounds of this sort are plagued by high costs and/or a lack of diversity in substitution patterns. Herein we report the conversion of the readily available bicyclo[1.1.1]pentan-1-amine substructure to a wide range of poly-substituted bicyclo[3.1.1]pentan-1-amines using imine photochemistry. To our knowledge, this is the first reported method to convert the bicyclo[1.1.1]pentane skeleton to the bicyclo[3.1.1]heptane skeleton. Hydrolysis of the imine products gives complex, $\mathrm{sp}^{3}$-rich primary amine building blocks.

MAIN TEXT: The fraction of carbon atoms that are $\mathrm{sp}^{3}$-hybridized in a drug candidate is positively correlated with the molecule's advancement though development and clinical trials. ${ }^{1}$ Growing cognizance of this trend among medicinal chemists has increased interest in building blocks containing small, bridged bicyclic carbon skeletons. These substructures, like aromatic rings, can provide a well-defined, rigid framework upon which to append substituents, but retain the pharmacological benefits of being aliphatic. ${ }^{2}$ However, most common building blocks containing such ring systems are mono- or "para"-disubstituted as a result of synthetic limitations. Consequently, they are restricted to use as linear spacers or as isosteric replacements for monosubstituted or paradisubstituted benzene rings. ${ }^{3}$ Furthermore, existing building blocks in this class are generally achiral, despite the positive correlation of stereochemical complexity with drug candidate success. ${ }^{4}$ The invention of new synthetic methods providing easy access to poly-substituted bicyclic cores will allow for a more complete biological evaluation of low molecular weight, $\mathrm{sp}^{3}$-rich chemical space.

To this end, our group has developed new methods to synthesize 1-aminonorbornanes, potential aniline bioisosteres with a high degree of substitutional variability. In particular, our group has reported an iridiumcatalyzed photoredox strategy and a metal-free, imine-based photochemical strategy toward these structures (Figure 1). ${ }^{5,6}$ In both cases, a species with $N$-centered radical character facilitates the homolytic cleavage of a bond in an adjacent cyclopropane ring, triggering an intramolecular radical ring closure cascade. In the photoredox case, the initial $N$-centered radical species is an amine radical cation, whereas in the imine-based case, it is the imine $\mathrm{S}_{1}\left(\mathrm{n}, \pi^{*}\right)$ excited state diyl. 


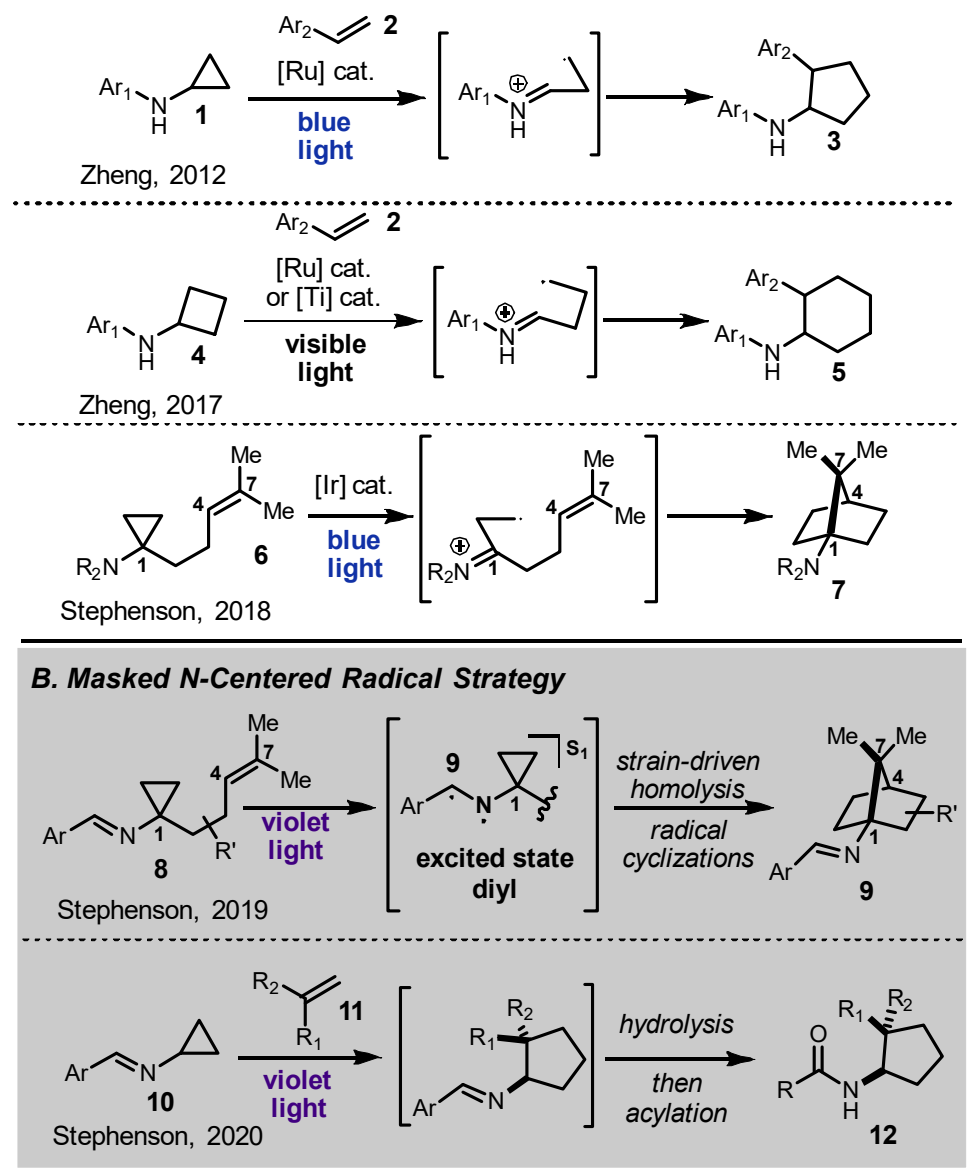

Figure 1. Selected inter- and intramolecular reactions featuring strain-driven homolysis of small-ring amines and subsequent ring formation via addition to an alkene

The photoredox strategy towards strain-driven homolysis and subsequent alkene addition is well-precendented. Zheng and coworkers developed intermolecular variants of the photoredox approach, reacting alkenes with cyclopropyl- or cyclobutylanilines to form 5- and 6-membered rings, respectively (Figure 1). ${ }^{\text {a-e }}$ This annulation chemistry was extended to cyclopropenes by Waser. ${ }^{8}$ However, the imine-based strategy had not been explored in the intermolecular context. Our group has recently disclosed work in this area. ${ }^{9}$ Though cyclopropane ring opening was observed in all cases, we have not observed cyclobutane ring opening under these conditions. This is consistent with the approximately $10^{3}$ difference in rate constants determined by Newcomb for the ring opening of cyclopropylaminyl radicals and cyclobutylaminyl radicals. ${ }^{10}$ Thus, we propose that the necessary imine excited state lifetime is sufficiently long to achieve cyclopropane ring opening but too short to realize the analogous reactivity with cyclobutanes. 
Previous kinetic studies
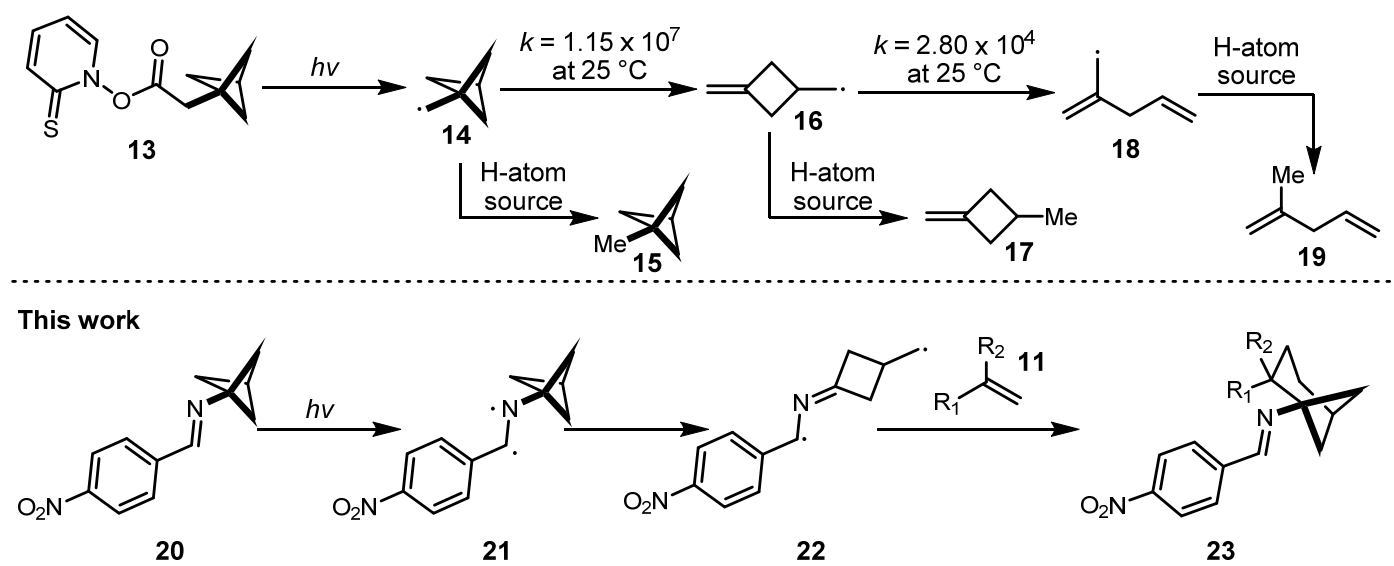

Figure 2. Rate constants from published studies of the bicyclo[1.1.1]pentylcarbinyl radical and our idea of trapping of the intermediate primary radical with an alkene

Intrigued by this difference in selectivity from that observed in the photoredox-based methods, we hypothesized that an imine derived from an abnormally reactive cyclobutylamine, namely a bicyclo[1.1.1]pentan-1-amine, may undergo the desired ring cleavage, generating primary radical intermediate 22, and then react with an alkene to afford an inert cyclobutylamine, namely a bicyclo[3.1.1] heptan-1-amine like 23. Though the risk of a second ring opening of the intermediate radical 22 was apparent, previous kinetic studies suggested that radicals of this sort (16) are long-lived enough to participate in intermolecular chemistry (Figure 2). ${ }^{11,12}$

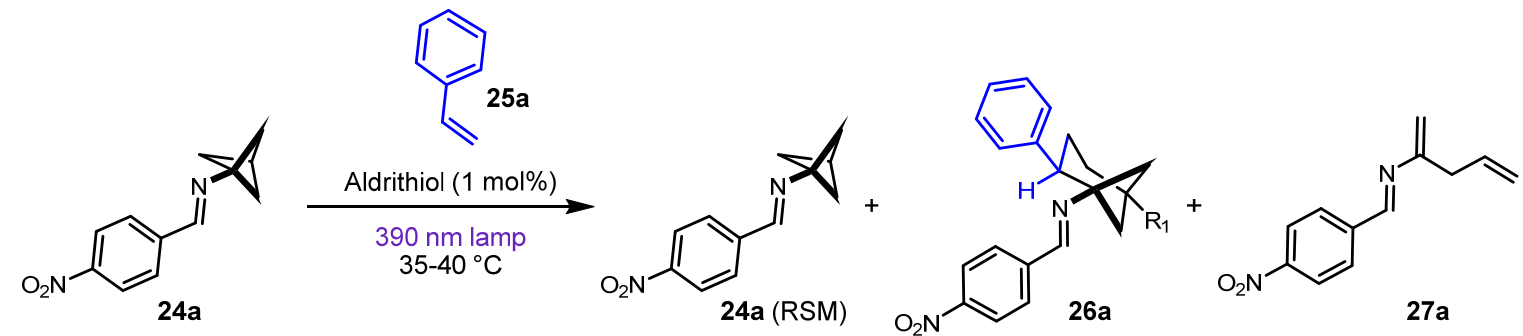

\begin{tabular}{|c|c|c|c|c|c|c|}
\hline Trial & 24a (mmol) & $\begin{array}{c}\text { Styrene } \\
\text { 2a (equiv) }\end{array}$ & Solvent & 24a (RSM) & 26a & 27a \\
\hline 1 & $0.2 \mathrm{mmol}$ & 7.5 equiv & $0.65 \mathrm{~mL} \mathrm{EtOAc}$ & $6 \%$ & $15 \%$ & $59 \%$ \\
2 & $0.2 \mathrm{mmol}$ & 7.5 equiv & $0.65 \mathrm{~mL} \mathrm{CH}{ }_{3} \mathrm{CN}$ & $5 \%$ & $16 \%$ & $57 \%$ \\
3 & $0.2 \mathrm{mmol}$ & 7.5 equiv & $0.172 \mathrm{~mL} \mathrm{EtOAc}$ & $7 \%$ & $29 \%$ & $40 \%$ \\
4 & $0.2 \mathrm{mmol}$ & 7.5 equiv & $0.172 \mathrm{~mL} \mathrm{CH} \mathrm{CHN}_{3}$ & $6 \%$ & $26 \%$ & $37 \%$ \\
5 & $0.4 \mathrm{mmol}$ & 7.5 equiv & neat & $18 \%$ & $38 \%$ & $23 \%$ \\
6 & $0.2 \mathrm{mmol}$ & 15 equiv & neat & $6 \%$ & $47 \%$ & $28 \%$ \\
\hline
\end{tabular}

Table 1: Optimization of reaction of the parent imine 1a and styrene 2a. Yields were determined by ${ }^{1} \mathrm{H}$ NMR spectroscopy of the crude reaction mixtures using 1,3,5-trimethoxybenzene as internal standard. RSM: recovered starting material. 
Indeed, irradiating 24a and styrene with $390 \mathrm{~nm}$ light in ethyl acetate gave the desired product 26a (Table 1, Trial 1). However, 26a was the minor product of the reaction, and skipped diene 27a, which results from the undesired background reaction, was the major product. Changing the solvent from ethyl acetate to acetonitrile had little effect on the reaction profile (Table 1, Trial 2). Increasing the concentration led to a drastic improvement in yield and selectivity, comparable for ethyl acetate and acetonitrile (Table 1, Trials 3 and 4). However, running the reaction in neat styrene gave the best results. Even in 7.5 equivalents of styrene, the imine dissolved, and the reaction proceeded to high conversion within 3 hours. The selectivity was comparable to when using 15 equivalents of styrene (Table 1, Trials 5 and 6). As a precaution, the additive Aldrithiol (2,2'-dipyridyl disulfide) was included in all cases since it was shown to reduce alkene polymerization in our intermolecular $[3+2]$ work. ${ }^{9}$

Despite the inclusion of the additive, prolonged reaction times led to an increasing amount of colored impurities and solid deposits inside the reaction vessel. However, these reaction times also sometimes led to decomposition of 27a through an unknown mechanism, thereby simplifying purification of the desired products. Reaction times of approximately 12 hours typically led to complete conversion and an acceptable impurity profile.

With suitable conditions in hand, the reaction scope was studied using the parent "para"-unsubstituted imine as well as the corresponding imine bearing a "para" methyl ester group (Figure 3). Gratifyingly, a wide range of styrenes showed the desired reactivity, albeit with modest yields. Reaction of $\mathbf{2 4 \mathbf { b }}$ with styrene gave product $\mathbf{2 6 \mathbf { b }}$ in $41 \%$ yield on a $10 \mathrm{mmol}$ scale (1.56 g product). Alpha-methyl styrene was well-tolerated, giving $\mathbf{2 6 g}$ and $\mathbf{2 6 h}$ in 32 and 30\% yields, respectively. 1,1-Diarylethylenes were able to undergo the desired ring closure to form sterically hindered bicyclo[3.1.1]heptan-1-amines but also produced a significant amount of a cyclobutenecontaining class of byproducts. Cyclobutene $\mathbf{2 8 I}$ was isolated in $11 \%$ yield alongside a 31\% yield of compound 261. We propose this byproduct forms via an intramolecular hydrogen atom abstraction proceeding through a 6membered cyclic transition state (Figure 4). Cyclobutenes of this type (31) and the ketones resulting from their hydrolysis (32) were observed as byproducts for several other alkene substrate classes. A number of examples of such byproducts were isolated and characterized. ${ }^{13}$ 

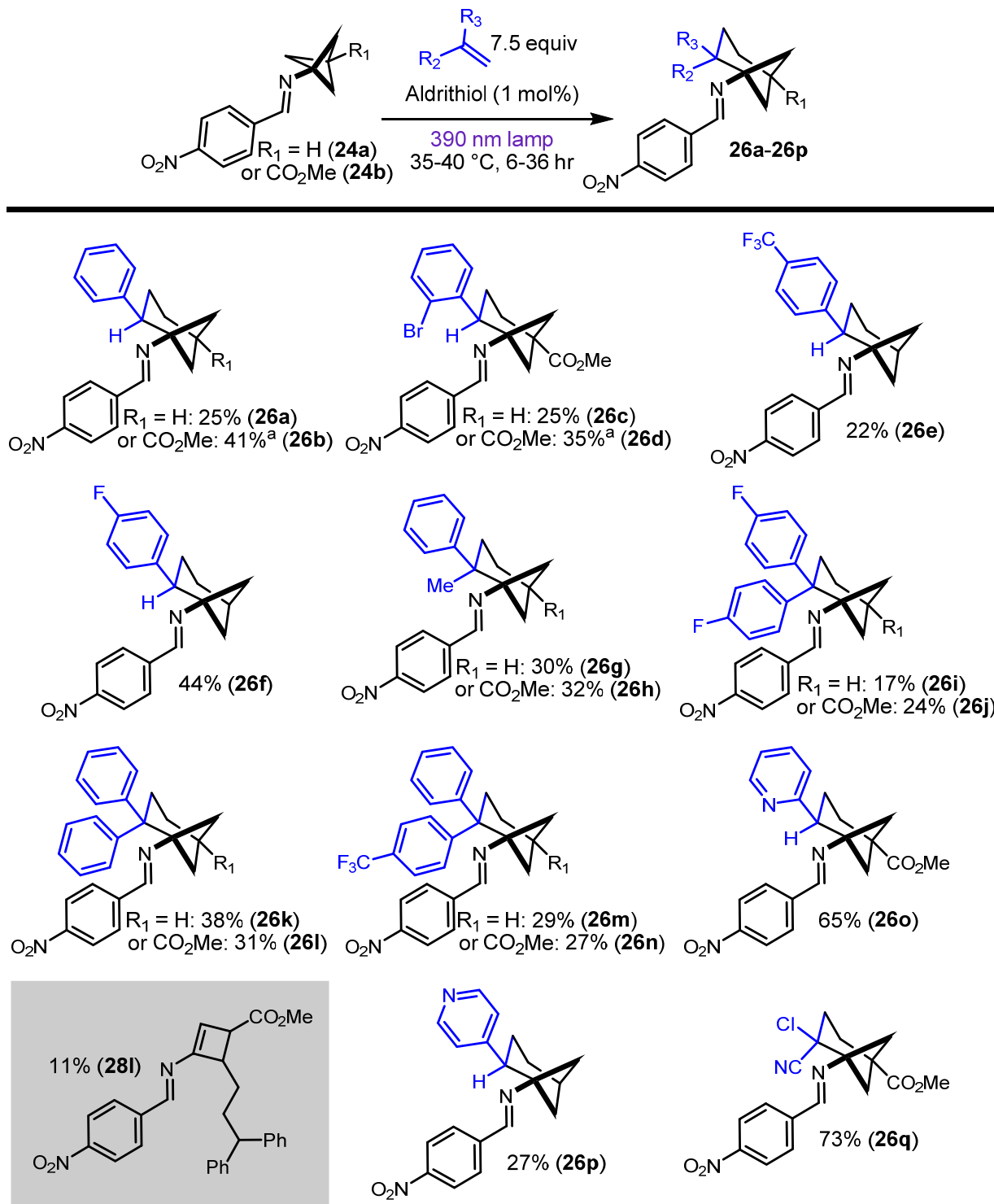

Figure 3. Examination of reaction scope. Standard reaction conditions: Imine (0.4 $\mathrm{mmol})$, alkene $(3.0 \mathrm{mmol}), 2,2^{\prime}$-dipyridyl disulfide $(0.004 \mathrm{mmol})$ in 1-dram vial with Teflon septum. Degassed through 3 freeze-pump-thaw cycles. Sealed with Parafilm ${ }^{\mathrm{TM}}$ and irradiated. Isolated yields reported. Deviations from standard reaction conditions: a) Performed on $10 \mathrm{mmol}$ scale in 6-dram vial. External temperature stabilized at $50{ }^{\circ} \mathrm{C}$ as measured by an infrared thermometer. Monitored by ${ }^{1} \mathrm{H}$ NMR. Starting material consumed after 36 hours. b) Two identical $0.4 \mathrm{mmol}$ runs were combined for purification. 
We were also pleased to see alkene classes besides styrenes undergo this chemistry. For example, vinyl pyridines gave modest to moderate yields of the desired products (Figure 3, Compounds 26n and 26o). Pyridines commonly act as H-bond acceptors in drugs and their incorporation into building blocks is appealing to for medicinal chemistry applications. Certain unsaturated carboxylic acid derivatives also participate in this reaction. Reaction of 24b with 2-chloroacrylonitrile gave compound $\mathbf{2 6 q}$ in $73 \%$ yield, the best performance we have observed yet for an alkene undergoing this reaction. Furthermore, the background reaction (Figure 4, "Undesired Reaction Pathway One") was completely suppressed by 2-chloroacrylonitrile, suggesting that certain alkenes may be reactive enough to compete with the background reaction even in the solution phase.
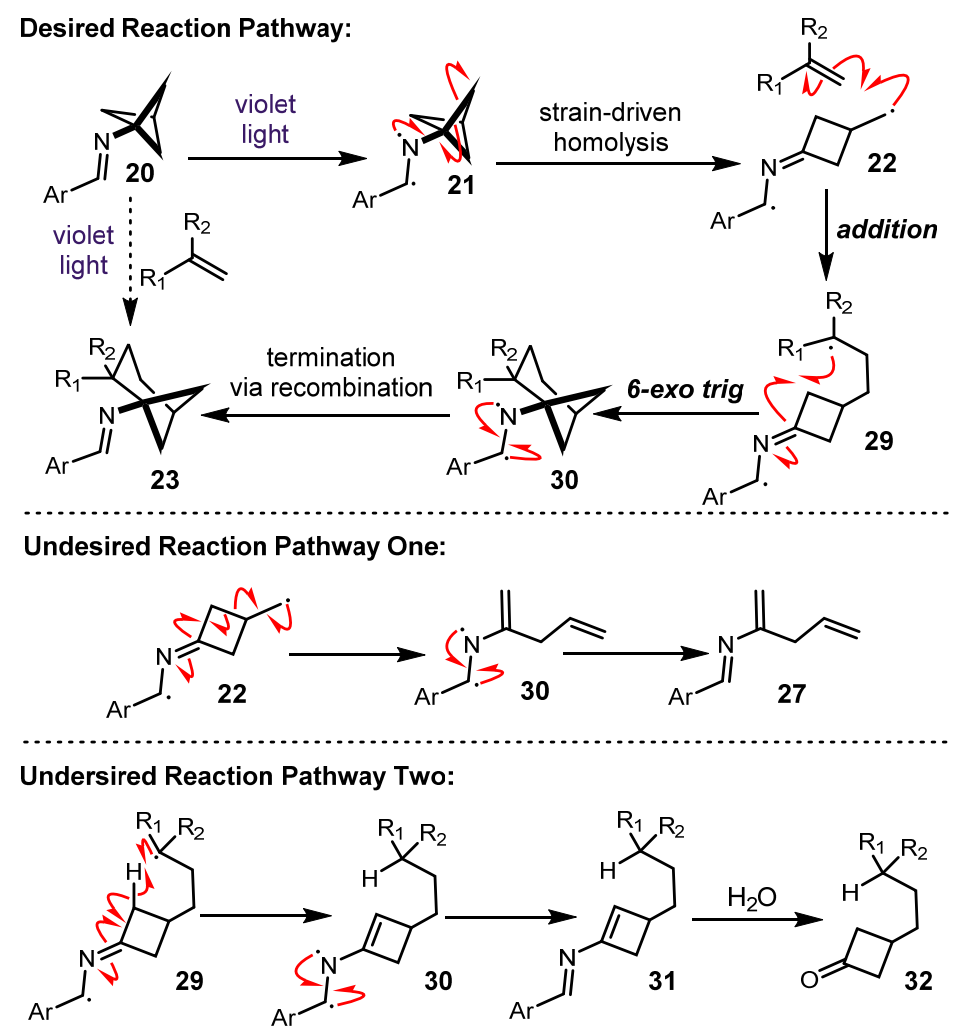

Figure 4. Proposed mechanisms for the formation of the desired product and the observed byproducts.

Imine 26b was elaborated to desirable protected gamma amino acid building blocks (Figure 5). First, 26b was hydrolyzed in $83 \%$ yield to give 33b. This material can be protected with di-tert-butyl dicarbonate to give carbamate $\mathbf{3 4 b}$, which in turn can be saponified to give amino acid building block $\mathbf{3 5 b}$ containing a protected amine and free carboxylic acid. Amine $\mathbf{3 3 b}$ can also be protected with benzyl chloroformate and subsequently saponified to give building block $\mathbf{3 6 b}$. 

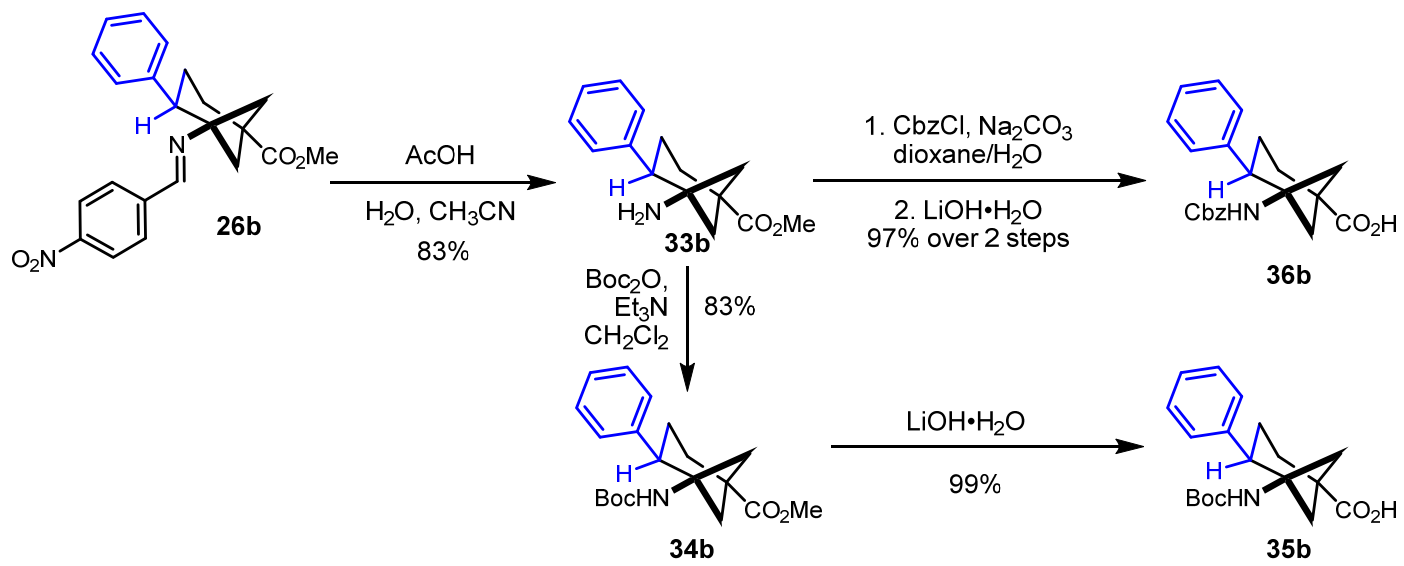

Figure 5. Conversion of $\mathbf{2 6 \mathbf { b }}$ to several protected gamma-amino acid building blocks.

In conclusion, we have developed a method of upgrading bicyclo[1.1.1]pentan-1-amines, an increasingly popular class of building blocks, to bicyclo[3.1.1] heptan-1-amines using imine photochemistry. Several classes of alkenes known to react rapidly with nucleophilic radicals are competent in this transformation. The imine products are easily hydrolyzed to give primary amine building blocks. Efforts are underway in our laboratory to further examine the scope of this reaction.

ACKNOWLEDGEMENTS: The authors acknowledge the financial support for this research from the NIH NIGMS (R01-GM127774) and the University of Michigan. MJS was supported by the Australian Governments Endeavour Leadership Fellowship. The authors thank Dr. Daryl Staveness (ExciPlex, Inc.) and Dr. Michael Harmata (University of Missouri) for helpful discussions.

\section{REFERENCES:}

1. Lovering, F.; Bikker, J.; Humblet, C. Escape from Flatland: Increasing Saturation as an Approach to Improving Clinical Success. J. Med. Chem. 2009, 52, 6752-6756.

2. Locke, G. M.; Bernhard, S. S. R.; Senge, M. O. Nonconjugated Hydrocarbons as Rigid-Linear Motifs: Isosteres for Material Sciences and Bioorganic and Medicinal Chemistry. Chem. Eur. J. 2019, 25, 45904647.

3. Mykhailiuk, P. K. Saturated bioisosteres of benzene: where to go next? Org. Biomol. Chem. 2019, 17, 2839-2849.

4. Lovering, F. Escape from Flatland 2: complexity and promiscuity. Med. Chem. Commun. 2013, 4, 515519. 
5. Staveness, D.; Sodano, T. M.; Li, K. Burnham, E. A.; Jackson, K. D.; Stephenson, C. R. J. Providing a New Aniline Bioisostere through the Photochemical Production of 1-Aminonorbornanes. Chem 2019, 5 , 215-226.

6. Staveness, D.; Collins III, J. L.; McAtee, R. C.; Stephenson, C. R. J. Exploiting Imine Photochemistry for Masked N-Centered Radical Reactivity.

7. (a) A relevant review: Hu, J.; Wang, J.; Nguyen, T.; Zheng, N. The chemistry of amine radical cations produced by visible light photoredox catalysis. Beilstein J. Org. Chem. 2013, 9, 1977-2001. (b) Wang, Q. Zheng, N. A Photocatalyzed Synthesis of Naphthalenes by Using Aniline as a Traceless Directing Group in [4 + 2] Annulation of Aminobenzocyclobutenes with Alkynes. ACS Catal. 2017, 7, 4197-4201. (c) Maity, S.; Zhu, M.; Sinabery, R. S.; Zheng, N. Intermolecular [3+2] Cycloaddition of Cyclopropylamines with Olefins by Visible-Light Photocatalysis. Angew. Chem. Int. Ed. 2012, 51, 222-226. (d) Wang, J.; Zheng, N. The Cleavage of a C-C Bond in Cyclobutylanilines by Visible-Light Photoredox Catalysis: Development of a [4+2] Annulation Method. Angew. Chem. Int. Ed. 2015, 54, 11424-11427. (e) Wang, J.; Mao, C.; Feng, P.; Zheng, N. Visible-Light-Mediated [4+2] Annulation of N-Cyclobutylanilines with Alkynes Catalyzed by Self-Doped $\mathrm{Ti}^{3+} @ \mathrm{TiO}_{2}$.Chem. Eur. J. 2017, 23, 15396-15403.

8. Muriel, B.; Gagnebin, A.; Waser, J. Synthesis of bicyclo[3.1.0]hexanes by (3+2) annulation of cyclopropenes with aminocyclopropanes. Chem. Sci. 2019, 10, 10716-10722.

9. Sowden, M.; L. Collins III, J.; Staveness, D.; Stephenson, C. R. J. A One Pot Photochemical Method for the Generation of Functionalized Aminocyclopentanes, 2020. https://doi.org/10.26434/chemrxiv.13079159.v1.

10. Musa, O. M.; Horner, J. H., Shahin, H.; Newcomb, H. A Kinetic Scale for Dialkylaminyl Radical Reactions. J. Am. Chem. Soc. 1996, 118, 3862-3868.

11. Walton, J. C. 3-Methylenecyclobutyl, Cyclopent-3-eny1, and 3-Methylenecyclobutylmethyl Radicals; Absence of Homoallylic Conjugation. J. Chem. Soc. Perkins. Trans. 2 1987, 231-235.

12. Branchaud, B. P.; Glenn, A. G.; Stiasny, H. C. Round-Trip Radical Probes: Ring Cleavage of the Bicyclo[1.1.1]pentylcarbinyl Radical. J. Org. Chem. 1991, 56, 6656-6659.

13. See the Supporting Information for further information on byproducts and their characterization. 